\title{
La revolución del blended learning en la educación a distancia
}

\section{The blended learning revolution in distance education}

\author{
Rosa García-Ruiz \\ Universidad de Cantabria, UC (España) \\ Ignacio Aguaded \\ Universidad de Huelva, UHU (España) \\ Antonio Bartolomé-Pina \\ Universitat de Barcelona, UB (España) \\ Coordinadores del Monográfico
}

\section{Resumen}

Los avances tecnológicos adaptados al ámbito educativo han favorecido que la educación a distancia sea una opción formativa con un aumento significativo en el número de instituciones y de estudiantes que optan por esta modalidad. Evidentemente la rápida y continua aparición de recursos tecnológicos que se están integrando en el contexto educativo en todos los rincones del mundo supone un esfuerzo constante en su adaptación para lograr que el proceso de enseñanza-aprendizaje sea eficaz y adecuado a los nuevos contextos. El blended learning se consolida como una modalidad educativa que está revolucionando la educación a distancia y que se ha enriquecido con nuevos recursos tecnológicos e innovadoras propuestas metodológicas, superando obstáculos y avanzando en nuevas posibilidades que garantizan la calidad de la educación semipresencial. En este sentido, se presentan en este trabajo las aportaciones de expertos internacionales en blended learning que, a partir de un conocimiento y análisis profundo de la literatura científica y de las investigaciones focalizadas en esta modalidad de enseñanza, ofrecen experiencias y prácticas enriquecidas con tecnologías y metodologías emergentes como la realidad aumentada, las rúbricas electrónicas, la ludificación o el aula inversa, entre otras, que plantean una modalidad educativa adaptada a las necesidades de un profesorado que busca nuevas formas de lograr la eficacia en su actividad docente y de un alumnado que espera de las instituciones educativas respuestas adecuadas a sus necesidades de formación.

Palabras clave: enseñanza semipresencial; innovación educativa; educación a distancia; aprendizaje; modelos de enseñanza. 


\begin{abstract}
The technological advances undertaken in the educational field have promoted distance education as an option with a significant increase in the number of institutions and students that opt for this modality. Evidently, the rapid and continuous appearance of technological resources that are being integrated into the educational context in all corners of the world implies a constant effort in its adaptation to ensure that the teaching-learning process is effective and adequate to the new contexts. Blended learning has been consolidated as an educational modality that is revolutionizing distance education and has been enriched with new technological resources and innovative methodological proposals, overcoming obstacles and making progress in new possibilities that guarantee the quality of blended education. In this sense, the contributions of international experts on blended learning are presented in this work. These contributions, which are based on in-depth knowledge and analysis of the scientific literature and the research focused on this teaching modality, offer experiences and practices enriched with technologies and emerging methodologies such as augmented reality, electronic rubrics, gamification and the flipped classroom, among others. These experiences and practices propose an educational modality adapted to the needs of a teacher who seeks new ways of achieving efficiency in their teaching activity and of a student body that expects educational institutions to respond appropriately to their training needs.
\end{abstract}

Keywords: blended learning; educational innovation; distance education; learning; teaching models.

En estos últimos años, de profundos avances tecnológicos y de la universalización de los dispositivos, constatar fehacientemente que la enseñanza a distancia está alcanzando altas cotas de éxito, no solo en su calidad didáctica, sino también en la satisfacción de los usuarios, podría suponer que se priorizara sobre otras modalidades de enseñanza más vinculadas al modelo educativo tradicional, totalmente presencial, o a la combinación de ambas. En este contexto, el blended learning ha ido progresivamente ganando terreno y "normalizándose", posicionándose como una modalidad didáctica para enseñar y para aprender, más pertinente que nunca, avalada por significativas experiencias y ya con un amplio y sólido recorrido, con una oferta formativa amplia, atractiva y cada vez más demandada.

El blended learning se ha constituido, a su vez, en un campo de estudio, en un ámbito de investigación científica, en la avanzadilla de la experimentación, que trata de hallar herramientas, recursos y perspectivas novedosas que potencien la enseñanza y minimicen los problemas del aprendizaje en una sociedad mediatizada como la que vivimos. Por ello, la investigación se revela como esencial para, en este entorno totalmente novedoso, minimizar las dificultades encontradas en su implementación y aportar nuevas estrategias y entornos en los que demostrar su eficacia educativa, tratando de mejorar su estructura, adaptarse a los nuevos roles de los profesores y estudiantes implicados, a innovadores contextos y recursos digitales. 
En consecuencia, nos encontramos ante un panorama presente y futuro en el que el blended learning comienza a ser una modalidad de enseñanza "normalizada", con sus peculiaridades específicas y su potencialidad "sui generis", capaz de adaptarse cada vez mejor a las necesidades del estudiante, de personalizar el proceso instructivo para lograr un aprendizaje más activo, en un contexto didáctico cada vez más flexible y personalizado, y todo ello en entornos de aprendizaje virtuales y presenciales combinados. Sin embargo, hemos de ser conscientes también de las dificultades de estos procesos de enseñanza y aprendizaje y en algunos casos el problema de la motivación y las altas tasas de deserción, en muchos casos ajenos al proceso en sí y más vinculadas en las situaciones personales de docentes y discentes, pero que, no cabe duda, han de ser estudiadas para nuevas estrategias de interacción que refuercen el proceso docente-discente y potencien, tanto la interacción didáctica como los niveles de egreso.

En los últimos años, se observa fácilmente una profunda evolución en las concepciones y usos en blended learning. Tanto los usuarios (profesores y alumnos) como los expertos en desarrollo y didáctica, han ido evolucionando al compás de la irrupción de nuevas tecnologías emergentes adaptadas al ámbito educativo. La popularización de Internet, en primer lugar, y de las redes sociales posteriormente, y sobre todo el acceso universal, asequible y accesible a los dispositivos móviles (tablets y smartphones) ha permitido un cambio sustancial en la forma de concebir e implementar los modelos didácticos. Por primera vez el sistema de enseñanza tradicional, que se ha mantenido siglos casi inalterable, se tambalea como única y monolítica forma de aprendizaje, teniendo que compartir con otras modalidades más abiertas y flexibles, también más experimentales y, a veces fugaces. Los nuevos modelos están rompiendo los marcos espacio-temporales de siglos de enseñanza y ofrecen nuevas dinámicas de aprendizaje. No cabe duda que usuarios (docentes y alumnos) expertos diseñadores y también los investigadores, tienen ante sí un gran reto, fruto de la novedad y la intensa adaptación y flexibilidad que el blended learning exige y demanda.

Por ello, la oportunidad de este monográfico en RIED es más que evidente. Algunos de los estudios que ofrecemos avanzan en la reflexión sobre estas temáticas novedosas y originales. Conocer la capacidad de adaptación a innovaciones vinculadas al ámbito metodológico, a través del "flipped classroom", o a la aplicación de nuevos recursos tecnológicos como es la realidad aumentada, la impresión en $3 \mathrm{D}$ o la robótica... sin duda, ofrecen a profesores y estudiantes nuevas perspectivas de enseñanza y aprendizaje que trastocan los modelos tradicionales mediante nuevos medios y recursos para interactuar, colaborar, apropiarse de nuevas formas de aprender adaptadas a estrategias didácticas innovadoras. La revolución del blended learning no ha hecho más que comenzar.

Abre el número el artículo que lleva por título "Blended learning: panorama y perspectivas" y que muestra el panorama y la trayectoria del blended learning a partir de un estudio riguroso basado en la literatura científica de expertos de 
todo el mundo que han publicado sus trabajos en las bases de datos de revistas científicas más prestigiosas, en el que Antonio Bartolomé, Rosa García-Ruiz e Ignacio Aguaded analizan la evolución y repercusión conceptual de esta modalidad de enseñanza y detallan las líneas de investigación que, desde múltiples disciplinas y perspectivas, demuestran, a partir de buenas prácticas y evidencias empíricas, su potencial y validez, llegando a plantear las perspectivas de futuro del blended learning, vinculadas a sus grandes aliados, los nuevos medios interactivos y digitales y augurando una trayectoria exitosa.

El éxito del blended learning se ve reforzado con la utilización de recursos tecnológicos como la Realidad Aumentada. Julio Cabero y Verónica Marín en su artículo "Blended Learning y Realidad Aumentada: experiencias de diseño docente" nos muestran las posibilidades que esta tecnología ofrece a la enseñanza a distancia, a partir de una experiencia educativa en titulaciones universitarias. Los resultados de su implementación demuestran que el uso de Realidad Aumentada en esta modalidad educativa permite diseñar entornos educativos de calidad que, si bien requieren por parte del profesorado cierta creatividad y dominio de la tecnología para la creación de objetos de realidad aumentada, las mejoras en el aprendizaje del estudiante son muy significativas.

El tercer artículo titulado "Impacto de una rúbrica electrónica de argumentación científica en la metodología Blended-Learning" presenta una investigación realizada por Daniel Cebrián Robles, Manuel Cebrián de la Serna, María Jesús Gallego Arrufat y Jesús Quintana Contreras, en la que se pone de manifiesto el éxito de la combinación de estos dos enfoques, evidenciando que, tras la puesta en práctica de una experiencia didáctica en un grupo de estudiantes universitarios, el resultado constata que se mejoran las calificaciones de los estudiantes, así como su capacidad de argumentación, por lo que se demuestra su eficacia para la formación blended learning.

Ángel Torres-Toukoumidis, Luis Miguel Romero-Rodríguez y $\mathrm{M}^{\mathrm{a}}$ Amor PérezRodríguez presentan en el artículo que lleva por título "Ludificación y sus posibilidades en el entorno de blended learning: revisión documental" una novedosa aportación al blended learning poniendo en valor las virtudes de la ludificación en el ámbito de la modalidad semipresencial. A partir del estudio de investigaciones realizadas en múltiples contextos educativos, se concluye que los beneficios de la implementación de la ludificación en esta modalidad educativa suponen una mejora de la motivación de los estudiantes, los resultados de aprendizaje y el desarrollo competencial.

El quinto artículo que conforma este monográfico lleva por título "Análisis cuantitativo y cualitativo de la semipresencialidad del sistema universitario de Cataluña”. Sus autores, Juan Simon Pallisé, Carles Benedí González, Cèsar Blanché i Verges, Maria Bosch i Daniel y Mercedes Torrado Fonseca, presentan una interesante perspectiva de las diversas tipologías del blended learning o Aprendizaje Combinado en las 12 universidades catalanas en las que se ha basado 
la investigación, destacando su limitada implantación en los planes de estudio, especialmente en las universidades públicas más grandes. Si bien, existen algunos casos en los que se ofrece esta modalidad de formación con buenos resultados. Los autores ofrecen una serie de sugerencias para garantizar el éxito de la implantación de la enseñanza Blended Learning que sin duda, no dejarán indiferente al lector.

Carlos Castaño Garrido, Urtza Garay Ruiz y Sotiris Themistokleous destacan en su artículo "De la revolución del software a la del hardware en educación superior", una visión tremendamente positiva de las posibilidades del blended learning a partir de un exhaustivo análisis de las posibilidades que ofrecen los nuevos escenarios de aprendizaje híbridos, combinados con nuevas metodologías de aprendizaje, apoyadas a su vez en tecnologías derivadas de la Web 2.o, capaces de propiciar no solo el trabajo colaborativo para crear conocimiento, sino que a través de la revolución que supone el software, se superan los límites y dificultades de la formación virtual, favoreciendo la explosión de una cultura "maker" y la creación de espacios "FabLab". Se necesitan hacer cambios importantes en las instituciones educativas para alcanzar el panorama presentado, pero en este trabajo se descubren algunas sendas por las que ir avanzando.

En el artículo titulado "Aportaciones de la formación Blended Learning al desarrollo profesional docente", sus autoras Ana Duarte Hueros, María Dolores Guzmán Franco y Carmen Rocío Yot Domínguez, a partir de una investigación documental en la que extrae información de artículos científicos vinculados a estos tópicos, plantean que esta modalidad formativa favorece el desarrollo de competencias docentes y a su vez el desarrollo profesional de este colectivo. Los resultados extraídos ponen de manifiesto que el blended learning es la modalidad de formación más pertinente para el profesorado, con una repercusión directa en las prácticas profesionales, aunque para que su aprovechamiento sea óptimo se destacan algunos requisitos fundamentales, como un diseño instruccional que englobe tanto la modalidad presencial como la on-line, que se favorezca la participación y colaboración social de los participantes y que la participación en esta formación esté apoyada por las propias instituciones educativas.

A partir de la aplicación práctica de una modalidad de blended learning en la que se utilizan la metodología de "aula inversa" o "flipped classroom" y la metodología "just in time teaching" (JiTT), Alfredo Prieto Martín, David Díaz Martin, Isabel Lara Aguilera, Jorge Monserrat Sanz, Paquita Sanvicen Torner, Raúl Santiago Campión, Alfredo Corell Almuzara y Melchor Álvarez-Mon Soto, en su artículo "Nuevas combinaciones de aula inversa con just in time teaching y análisis de respuestas de los alumnos", ofrecen los resultados de una experiencia llevada a cabo en el ámbito universitario, los cuales indican que el rendimiento académico de los estudiantes ha mejorado, y que las valoraciones de los estudiantes avalan la eficacia de esa modalidad combinada. Sin duda, nos encontramos con un modelo de buenas prácticas que presenta una interesante propuesta de mejora del aprendizaje de los estudiantes con información contrastada y aplicable a otros contextos que 
garantizarán la eficacia de la metodología de aula invertida respecto a la mejora del rendimiento académico.

El último manuscrito de este monográfico lleva por título "Blended Learning, más allá de la clase presencial”, en el que Jesús Salinas Ibáñez, Bárbara de Bemotp Crosetti, Adolfina Pérez Garcías, y Mercè Gisbert Cervera ofrecen una serie de recomendaciones, a partir del análisis de la literatura existente, que inciden en la necesaria flexibilidad y adaptación de las modalidades más adecuadas para lograr que el blended learning se posicione como una modalidad de formación adecuada para estudiantes universitarios y de otros niveles o estudios. Teniendo en cuenta las recomendaciones planteadas, este monográfico que RIED ofrece a los lectores es una magnífica oportunidad para conocer buenas prácticas en la implementación del blended learning en la docencia y para tener, así mismo, un conocimiento exhaustivo de las líneas de investigación que se han desarrollado hasta el momento en el panorama internacional en relación a esta temática, por lo que los profesores y expertos en blended learning tienen a su disposición información suficiente y contrastada para lograr dar respuestas a los retos que estos autores plantean.

Esta compilación de artículos de expertos y estudiosos del blended learning ofrece una perspectiva amplia y un profundo conocimiento de las posibilidades de esta modalidad educativa, de su trayectoria a partir de investigaciones científicas que han mostrado su eficacia, su evolución, y sus limitaciones, también a partir de experimentaciones reales, constatadas con rigurosidad y fundamentación metodológica. Sin duda, este monográfico ofrece a docentes y responsables de instituciones educativas una visión holística de las implicaciones didácticas de una modalidad de enseñanza aprendizaje que está apropiándose de las tecnologías emergentes para revolucionar un modelo educativo, trasnochado en la mayoría de las universidades, que debe necesariamente avanzar en la búsqueda de ofertas formativas adaptadas a los nuevos estudiantes, a los nuevos ciudadanos que demandan otra forma de aprender, de construir el conocimiento a partir de su interacción con la comunidad educativa, con el contexto digitalizado en el que vivimos. Sin duda, cada una de las aportaciones de este monográfico plantea algunas de las claves para un cambio de paradigma educativo necesario, esperado y cada vez más cercano, en el que el blended learning se posiciona como una modalidad de enseñanza indiscutiblemente potente, válida y transferible a todos los contextos.

\section{PERFIL ACADÉMICO Y PROFESIONAL DE LOS AUTORES}

Rosa García-Ruiz. Profesora del Departamento de Educación y de Universidad de Cantabria (España), adscrita al área de Didáctica y Organización Escolar. Editora adjunta de la Revista Comunicar y Editora jefa de la Revista Alteridad. Responsable del Grupo de Investigación en Educación Mediática de la Universidad de Cantabria (EDUCAMED), perteneciente a la Red de Excelencia en Educación Mediática (EDU2016-81772-REDT). Coordinadora de la Red 
Interuniversitaria Euroamericana ALFAMED-España. Es profesora colaboradora en el Máster Internacional de Comunicación y Educación Audiovisual (UNIA/UHU) y del Programa Interuniversitario de Doctorado en Comunicación (US, UMA, UCA y UHU).

E-mail: rosa.garcia@unican.es

\section{DIRECCIÓN DE LA AUTORA}

Universidad de Cantabria

Facultad de Educación

39005-Santander (Cantabria) - España.

Ignacio Aguaded. Catedrático de Universidad del Departamento de Educación de la Universidad de Huelva (España). Presidente del Grupo Comunicar, colectivo veterano en España en Educomunicación, y Editor de la Revista «Comunicar» (indexada en JCR, Scopus, ERIH, RECYT...). Es además Director del Grupo de Investigación "Ágora», responsable de múltiples investigaciones internacionales y de la dirección de numerosas tesis doctorales. Es Director del Máster Internacional de Comunicación y Educación Audiovisual (UNIA/UHU) y Subdirector del Programa Interuniversitario de Doctorado en Comunicación (US, UMA, UCA y UHU). Premio al Mejor Investigador de la Universidad de Huelva 2015 en Ciencias Sociales. Ha sido Vicerrector de Tecnologías, Innovación y Calidad de la Universidad de Huelva durante 7 años.

E-mail: aguaded@uhu.es

\section{DIRECCIÓN DEL AUTOR}

Universidad de Huelva

Facultad de Ciencias de la Educación

21071 Huelva (Spain)

Antonio Bartolomé-Pina. Doctor en Filosofía y Ciencias de la Educación. Ha trabajado en el diseño y desarrollo de sistemas multimedia para la Educación desde 1988, vídeo digital y entornos de aprendizaje potenciados por la tecnología. Dirige el Instituto de Investigación Educativa de la Universitat de Barcelona y actualmente centra su actividad investigadora en el aprendizaje personalizado con soporte digital y las posibilidades de las "blockchain" en Educación.

E-mail: abartolomepina@gmail.com 


\section{DIRECCIÓN DEL AUTOR}

Facultat d'Educació, Universitat de Barcelona

Pg. Vall Hebron, 171, Campus Mundet

o8035 Barcelona

\section{Como citar este artículo:}

García-Ruiz, R., Aguaded, I., y Bartolomé, A. (2017). La revolución del "blended learning" en la educación a distancia. RIED. Revista Iberoamericana de Educación a Distancia, 21(1), pp. 25-32. doi: http://dx.doi.org/10.5944/ried.21.1.19803 